\title{
Tuning characteristics of the top-down attentional setting
}

\author{
Fook K. Chua
}

Published online: 9 January 2013

(C) Psychonomic Society, Inc. 2012

\begin{abstract}
Previous studies of top-down attentional guidance have focused generally on the attentional prioritization of a single target feature. The present study focused on how the attentional system would be configured when the target possesses several unique features. These features were perfectly correlated, which meant that monitoring just one of them would be an adequate strategy. The experiments addressed the following questions: (a) If the target is a singleton, would the attentional system be set to monitor the target's unique features, or would the search strategy default to singleton detection? (b) Can the target's static and dynamic features be prioritized simultaneously? (c) Are all of the target's features prioritized, or only those features that are diagnostic of targethood? The results revealed an attentional system that can be flexibly tuned to multiple target features across static and dynamic dimensions. This system can simultaneously be narrowly tuned to monitor a specific target feature and broadly tuned to detect singletons. Finally, the attentional system monitors only those features that are diagnostic of targethood.
\end{abstract}

\section{Keywords Selective attention · Attentional capture}

Search for a salient object (e.g., a feature singleton among homogeneous distractors) is efficient, with search latencies roughly independent of display set size. This result has been extensively replicated and has formed the basis of many models of visual search (e.g., Treisman \& Gelade, 1980; Wolfe, Cave, \& Franzel, 1989), as well as of computational models of visual attention (e.g., Itti \& Koch, 2000). One widely held conception is that, at the preattentive stage, the

\section{F. K. Chua $(\square)$}

Department of Psychology, National University of Singapore,

Kent Ridge Crescent,

Singapore 119260, Singapore

e-mail: fkchua@nus.edu.sg saliency values of the visual scene are encoded in a twodimensional feature map. Fast, bottom-up mechanisms bias orienting toward the most salient location, and the saliency of a target that is a singleton will be high relative to the rest of the field. Thus, localizing the target is expected to be efficient because attention can be guided swiftly to its location, thereby allowing it to "pop out." The question is whether this constitutes evidence of bottom-up attentional guidance.

In the singleton-search experiment, saliency and targethood are perfectly correlated. However, for capture to be demonstrated unambiguously, it is essential that the experimental design ensure that the observer's goal does not entail seeking the salient stimulus: That is, targethood and saliency have to be decoupled. Yantis and Egeth (1999) showed that when the object's salient feature was not diagnostic of targethood, attention would not automatically be guided to it (but see Lamy \& Zoaris, 2009, for a recent reevaluation). There appears, however, to be an exception. Yantis and his colleagues (e.g., Jonides \& Yantis, 1988; Yantis \& Hillstrom, 1994; Yantis \& Jonides, 1984, 1996) reported that an object appearing abruptly constituted the only case of bottom-up attentional guidance. This claim was disputed by Folk, Remington, and their colleagues (Folk, Remington, \& Johnston, 1992; Folk, Remington, \& Wright, 1994), who argued that attentional orienting to an object is contingent on the observer's task-determined goals. Accordingly, unless the system has been specifically set to prioritize dynamic discontinuities, an abrupt onset would not capture attention. This proposal has been encapsulated in the contingent-involuntary-orienting hypothesis, in support of which Folk et al. (1992) showed that when the diagnostic target feature was not a dynamic discontinuity, an irrelevant onset cue failed to capture attention.

In the initial version of the contingent-orienting hypothesis, Folk et al. (1992) distinguished between static and dynamic discontinuities. The claim was that the attentional 
control setting (ACS) would be broadly tuned to respond to these discontinuities. For example, they demonstrated that when the diagnostic target was a red singleton, a nonmatching irrelevant green singleton cue succeeded in capturing attention, suggesting that a match between the target and the cue of the critical (color) dimension was not essential (Folk et al., 1992, Exp. 4). This claim converges with the proposal made by Bacon and Egeth (1994) that, when the search environment makes the target an oddball, the system defaults to a singleton-detection strategy.

More recent evidence, some of which has been inconsistent with the earlier results, has provided refinements to initial claims of a broadly tuned ACS. For example, Folk and Remington (1998) reported that the irrelevant color cue only succeeded in capturing attention when it matched the diagnostic target's color (see Folk \& Remington, 2008, for a replication), suggesting that the ACS had been tuned specifically to the target's color. Other results, however, suggest that the tuning function is not as narrow as was implied by Folk and Remington. Ansorge and Heumann (2003) demonstrated attentional orienting to a cue that did not precisely match the target's color. Anderson and Folk (2010) extended this finding, reporting a positive relationship between the magnitude of capture and cue-target similarity. Taken together, these results suggest that the tuning function is narrower than the initial Folk et al. (1992) conception, but broader than the Folk and Remington $(1998,2008)$ revision.

These new findings pose problems for Bacon and Egeth's (1994) proposal that an oddball target invites singleton detection as the default search strategy. Lamy, Carmel, Egeth, and Leber (2006) noted that results relating to the priming of pop-out (e.g., Maljkovic \& Nakayama, 1994) were largely incompatible with the Bacon and Egeth proposal. But, while these data spoke against the claim of a default singleton-detection strategy, they did not rule out singleton search as one of the options. Using the irrelevant-singleton paradigm, Lamy, Carmel, et al. contrasted a condition in which the target had a fixed diagnostic feature (fixed condition) with a condition in which the singleton's diagnostic feature assumed different values (mixed condition). Their findings have implications for the ACS's specifications. A comparison between the two conditions showed that the mixed condition had a significantly longer search latency and also a larger irrelevant-distractor effect. These two findings suggest that the ACS must have been programmed to respond specifically to the fixed target feature. But, crucially for the fixed condition, an irrelevant distractor still captured attention, which should not have occurred had the ACS merely been tuned narrowly to detect the specific target feature (as according to, e.g., Folk \& Remington, 1998, 2008). To resolve this inconsistency, Lamy, Carmel, et al. argued that the attentional system had been flexibly programmed to respond to a range of target- defining features. Thus, if the same oddball target was presented throughout the experiment, its singletonhood, as well as its unique feature, was diagnostic of targethood, and both of these sources of information would be deployed to guide attention.

The idea of a more flexibly programmed ACS finds support, in a different context, from Irons, Folk, and Remington (2012). They showed that the ACS may be tuned to monitor more than one value within a dimension. In their Experiment 1, the target varied unpredictably between one of two colors (e.g., red or green). An irrelevant color (e.g., blue) cue that did not match either of the two target colors nevertheless succeeded in capturing attention, suggesting a singleton-detection strategy. They foreclosed a singletondetection strategy in their Experiment 2 and ran the same irrelevant-color-cue conditions. The critical finding was that while there was attentional orienting to the color cues that matched either of the two target colors, the cue that matched neither of these colors failed to capture attention. The implication is that when warranted by task demands, the ACS may be narrowly tuned to two different hues.

The work on top-down attentional modulation has focused largely on a single feature from a single dimension (e.g., color). However, objects are constituted of more than one feature, and it is conceivable that a target may be unique on more than one dimension. The central issue of this set of experiments concerned how attention is guided to a target that is distinguished by more than one unique feature. The following scenario was adopted: The target was constituted of several perfectly correlated diagnostic features. Furthermore, the search environment was engineered to make the target a singleton. The question asked was, How might the top-down attentional setting be configured to locate the target swiftly and efficiently? Several solutions were possible. A target that is unique on several dimensions is likely to have its overall saliency enhanced. This might prompt the system to adopt saliency detection as the search strategy. The second possibility is that the ACS would be tuned narrowly to each of the target's values on the different dimensions. But, as these features were perfectly correlated, monitoring all of them would be superfluous, not to say hazardous. When attentional orienting could be triggered by any of several features, there could, potentially, be more false alarms. The third option is that the system monitors only the most salient of these target dimensions.

According to the contingent-orienting hypothesis (Folk et al., 1992, 1994), the ACS would be programmed to achieve the objective of expeditious localization of the target. The goal of the experiments in the present study was to reveal the nature of these attentional settings. The strategy that was adopted involved probing the attention-capturing capacity of an irrelevant cue presented before the search array was revealed. Across experiments, the featural overlap between 
this cue and the target was manipulated. If this specific target feature had been programmed into the ACS, there should be attentional orienting toward the cue. Otherwise, the cue would fail to capture attention. Thus, the pattern of attentional capture across these irrelevant cues would disclose the attentional prioritization scheme adopted by the system to facilitate target search.

\section{Experiment 1}

In Experiment 1, the target location was marked by both a static and a dynamic discontinuity. As the two discontinuities were perfectly correlated, monitoring one or the other would lead, unerringly, to the target. The question asked was whether the ACS would be set to monitor, simultaneously, both types of discontinuities. An irrelevant static or dynamic cue was presented before the search array. If both discontinuities had been prioritized, there should be orienting to both types of irrelevant cues.

\section{Method}

Observers For this and the other experiments in this study, the observers were recruited from the undergraduate subject pool. They participated in partial fulfillment of course requirements. All of the observers had normal or corrected-to-normal vision. A group of 15 observers took part in Experiment 1.

Procedure Figure 1 shows the sequence of frames presented in a typical trial. The objects that constituted the display were first ramped up (the first four frames in the left- and right-hand sequences, Fig. 1). Eight circles (radius $=0.5^{\circ}$, with the observers freely viewing the display from a distance of $50 \mathrm{~cm}$ from the screen) were arranged on the circumference of an invisible circle (radius $=4.5^{\circ}$ ), with the smaller circles being equidistant from each other. Four of the circles were filled, each with a digital " 8 " figure (Todd \& Van Gelder, 1979), and the other four circles were unfilled.

Two main variables were investigated. The first variable, the type of irrelevant cue, had two conditions. In the irrelevant-color-singleton condition, each of the four filled circles was initially surrounded by four spots. The objects all had the same luminance as they were ramped up, with the objects initially being invisible (background luminance $\sim 60 \mathrm{~cd} / \mathrm{m}^{2}$; CIE[Yxy]: $60.1, .31, .31$ ) and achieving their final luminance of $\sim 30 \mathrm{~cd} / \mathrm{m}^{2}$ in approximately $1 \mathrm{~s}$. After a pause of approximately $100-150 \mathrm{~ms}$, the irrelevant cue frame was presented: One set of gray spots turned red, and the other three sets turned blue (fifth frame of the left-hand sequence, Fig. 1). The color spots had the same luminance as the gray spots (red CIE[Yxy]: 30.1, .56, .29; blue CIE[Yxy]: 30.0, $.19, .17)$. In the irrelevant-onset condition, two of the unfilled circles were surrounded by spots as the display was ramped up. When the cue frame was presented, the spots turned blue, and simultaneously, an additional set of four blue spots appeared abruptly around one of the filled circles (fifth frame of the right-hand sequence, Fig. 1). Post-onset, this additional set of spots would not be considered a shape or a color singleton.

The cue frame was displayed for $200 \mathrm{~ms}$, following which the search array appeared. Several line segments were removed from each placeholder, revealing a camouflaged letter, and simultaneously, the spots surrounding the filled circles also disappeared. There were, therefore, offset transients at the four filled circles. In addition, all eight circles turned green (CIE[Yxy]: 29.8, .36, .52). At the target's location, an additional green ring appeared, surrounding the circle that enclosed the target letter (E or $\mathrm{H}$ ), creating a unique onset transient at that site. The two concentric circles enclosing the target constituted a shape singleton. The other, nontarget letters were selected from the set consisting of the following letters: A, P, S, and U. The observers' task was to identify the letter enclosed within the two concentric circles. The search array was presented for $200 \mathrm{~ms}$, after which the letters disappeared, leaving behind their circular surrounds.

The second variable was whether the irrelevant cue was valid, invalid, or neutral. In the valid condition, the location of the color singleton or onset cue was coincident with the location of the target letter (left-hand sequence, Fig. 1). In the invalid condition, a distractor letter was found inside the circle surrounded earlier by the irrelevant cue (right-hand sequence, Fig. 1). The ratio of valid- to invalid-cue trials was 1:3, making the cue unpredictive of the target location. Additionally, two neutral trials were presented for every four (valid and invalid) cue trials. For the neutral trials, the surrounding spots turned blue.

Two versions of the experiment were conducted. Version 1 (eight observers) was described above. The color singleton spots were red, and the nonsingleton spots were blue. In Version 2 (seven observers), the colors were reversed for the singleton and nonsingleton conditions. Summarizing, the design was a 2 (version) $\times 3$ (cue type) $\times 2$ (cue validity) factorial design. Each observer went through 13 blocks of trials, with each block consisting of 24 trials. The first block was regarded as a practice block, and its data were not analyzed.

The observers were told that some form of visual distraction would appear during the trial and were advised to keep their attention on the fixation cross. They were told explicitly that the target letter would be found within the only two concentric circles in the display; that is, the instructions emphasized the shape singleton enclosing the target letter. 
Fig. 1 Sequences of frames presented in typical irrelevantcolor-singleton and irrelevantonset cue conditions. Left sequence: The color singleton cue condition. Here, the objects were ramped up (first four frames). At some point, the (valid) color singleton was presented - one set of spots turned from gray to blue (white, in the figure above), and the other three sets turned red (gray in the figure). The surround spots then disappeared when the search array appeared, with an additional circle appearing around the circle enclosing the target. In the final frame, the letters disappeared, leaving the circular surrounds. Right sequence: Onset cue condition. The sequence was similar for the onset cue, except that a set of spots appeared around a filled ring when the (here, invalid) cue was presented

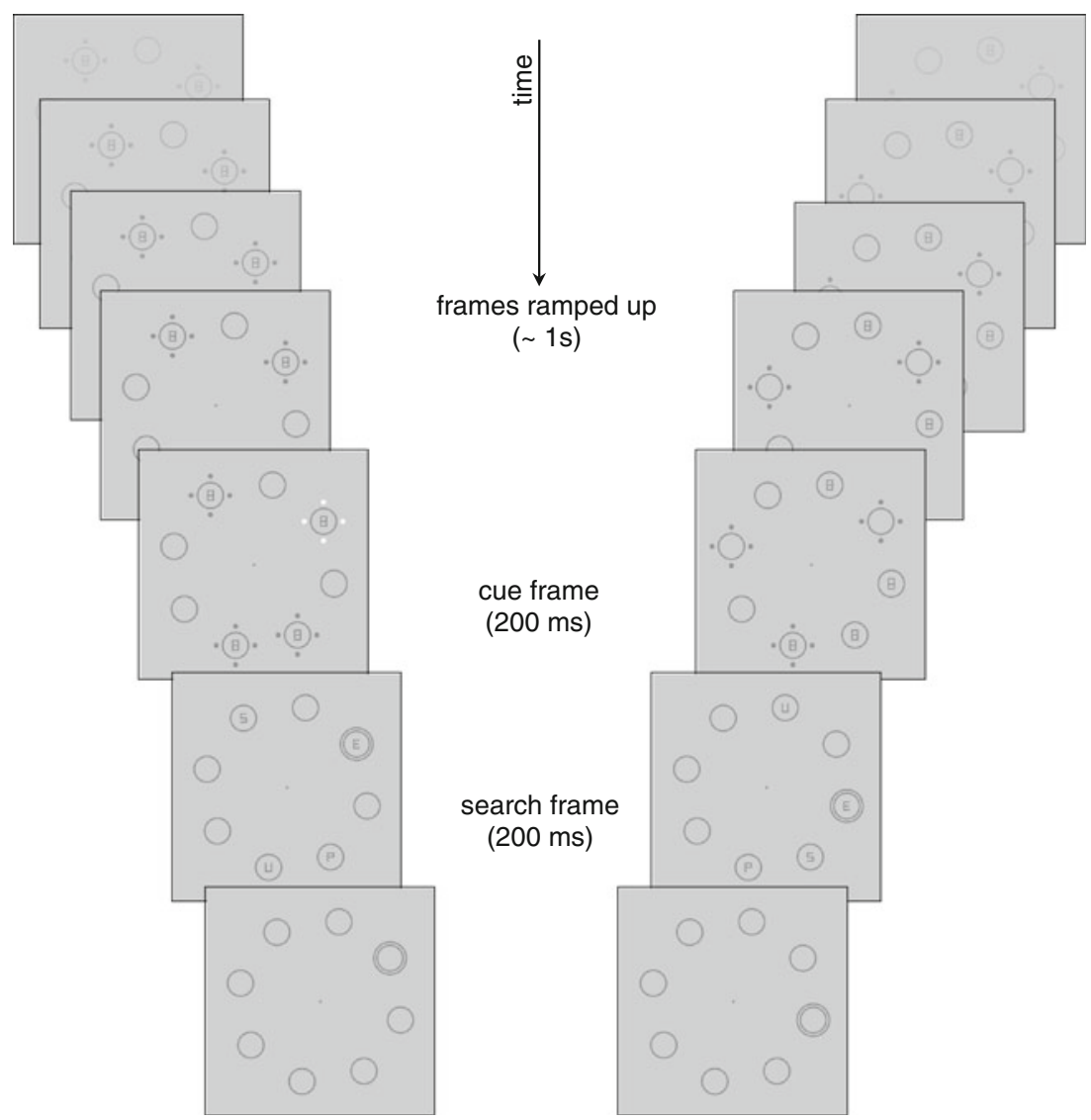

Results

The latency and error data for the various cue validity conditions, as a function of type of irrelevant cue, are presented in Fig. 2. Analyses of the data proceeded in two steps. First, the data were assessed for a speed-accuracy trade-off. In Experiment 1, the conditions in which response times (RTs) were longer were the same conditions in which error rates were higher, suggesting that a speed-accuracy trade-off was unlikely. This was confirmed in a formal analysis of the error data. ${ }^{1}$

The main analysis concerned the question of whether attention had been captured by the two types of irrelevant cues. The signature of attentional capture was shorter RTs in the valid, and longer RTs in the invalid, condition, with the

\footnotetext{
$\overline{{ }^{1} \text { The accuracy }}$ data were analyzed in a 2 (version) $\times 2$ (cue type) $\times 3$ (cue validity) ANOVA. No version effect emerged, nor did version interact with the other variables, $F \mathrm{~s}<1$. Furthermore, the cue type effect was not significant, $F<1$. The invalid-cue condition (which had a longer mean RT) was significantly less accurate than the valid-cue condition, $F(2,28)=6.020, p<.01$. The interaction effect was also significant, $F(2,28)=6.176, p<.01$. For the color singleton condition, the accuracies were comparable for the three validity conditions. However, for the onset condition, there was an inverse relationship between accuracy and latency.
}

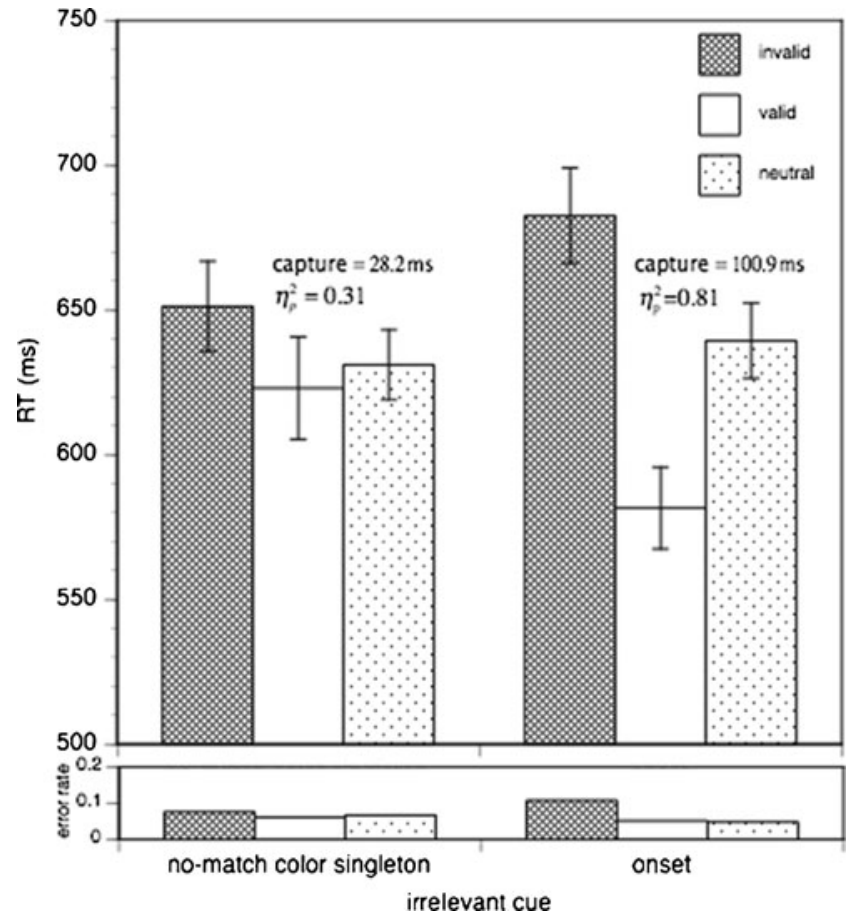

Fig. 2 Experiment 1: Mean response times (RTs) and error rates in the valid, invalid, and neutral cue conditions, as a function of type of irrelevant cue. The magnitude of the capture effect is computed by taking the difference between the invalid and valid cue conditions. The capture effect size is also reported. Error bars $=1 S E$ 
mean RT of the neutral condition lying between these two conditions. This pattern was observed for both types of cues.

A 2 (version) $\times 2$ (cue type: color singleton vs. onset) $\times 3$ (cue validity: valid, invalid, and neutral) mixed-factor analysis of variance (ANOVA), with Version treated as a between-subjects factor, revealed no version effect, nor any reliable interactions between it and the other variables, $F \mathrm{~s}<1$. Also, no overall effect of cue type appeared, $F<1$. However, the cue validity variable was significant, $F(2,28)=32.897, p<.001, \eta_{\mathrm{p}}{ }^{2}=.72$, as was the interaction between cue type and cue validity, $F(2,28)=11.067, p<.001, \eta_{\mathrm{p}}{ }^{2}=.47$. The significant interaction implies that the spatial-cuing effect was modulated by cue type. The cue validity effect $(101 \mathrm{~ms})$ in the onset-cue condition was more than three times the size of the effect $(28 \mathrm{~ms})$ in the color singleton condition. The latter effect was nonetheless reliable, $F(1,14)=$ 6.242, $p<.05, \eta_{\mathrm{p}}{ }^{2}=.31$.

These overall results are consistent with the hypothesis that the observers' ACSs had been tuned to monitor both the spatial and the dynamic discontinuities. Before this interpretation is considered further, it is important to rule out an alternative account: The observed effects were averaged across observers, so the same pattern would be obtained if some observers set their ACSs to monitor exclusively the spatial discontinuity, while others monitored only the dynamic discontinuity. According to this view, there should not be a correlation between the capture effects for the two irrelevant cues; that is, if capture was obtained by the onset cue, capture should not occur for the color cue, and vice versa. (It is assumed that the control settings were set at the start of, and maintained consistently throughout, the experiment.) Of the 15 observers, one observer showed negative effects (i.e., the mean RT was longer for the valid than for the invalid cue) for both cues. Two other observers showed a positive effect for the onset cue, but a negative effect for the color singleton condition. The rest of the 12 observers showed positive effects for both cues. A sign test of the data revealed a significant result $(p<.05)$, contradicting the hypothesis that observers had been monitoring either the spatial or the dynamic discontinuity, but not both.

\section{Discussion}

Two interesting results were obtained here: (a) Both the irrelevant dynamic and static cues captured attention, and (b) the capture effect was significantly larger for the dynamic than for the static cue.

The target location was marked by a shape singleton. The static discontinuity presented during the cue frame was a color singleton, making the setup in this experiment analogous to that of Theeuwes (1992), who enclosed the target in a shape singleton and, simultaneously, presented an irrelevant color singleton. He reported that the color singleton distractor impeded search, which he interpreted as an example of bottom-up capture by a salient (color) feature. A similar effect was found in Experiment 1: The irrelevant color cue presented before the search array affected search for a unique shape.

In considering Theeuwes's (1992) results, Bacon and Egeth (1994) observed that the singleton target may have prompted a singleton-detection strategy. According to this view, Theeuwes's (1992) results should not be regarded as an instance of bottom-up capture. Some recent data, however, have appeared to contradict the Bacon and Egeth claim that, so long as it is feasible, singleton detection would be adopted as the default search strategy (e.g., Folk \& Remington, 1998, 2008). In view of these new findings, how might one explain capture in the irrelevant-colorsingleton condition? One approach would be to follow Theeuwes's (1992) lead and appeal to the saliency of the color singleton cue.

There is an alternative explanation, however. Lamy et al. (2006b) reported data suggesting that singleton detection and feature search need not be mutually exclusive strategies, as both can be maintained simultaneously. Extending Lamy, Carmel, et al.'s observation to the context of this experiment, the ACS could have been set to monitor the specific target shape and onset transient and, as the target was an oddball, also, simultaneously, be set to detect singletons.

Experiment 1's results cannot distinguish between the saliency account and Lamy, et al.'s (2006) explanation. The data are, however, incompatible with an intertrialpriming account. The defining target features were a unique onset transient and a shape singleton. While attentional guidance to the onset cue might be attributed to priming, this explanation will not work for the color singleton cue condition, as its color did not appear in the search array. For the priming account to work, one would have to assume some form of interdimensional priming. Lamy, Bar-Anan, Egeth, and Carmel (2006, Exp. 5) ruled out this explanation.

The capture magnitude was weaker in the color cue condition than in the onset cue condition. Three explanations are proposed: (a) The color cue is inherently less salient than the onset cue, and the difference in capture magnitudes reflects this disparity. (b) According to Theeuwes's (2010) disengagement account, the duration of preattentive capture by a salient feature would be very brief if subsequent top-down attentional modulation did not follow. This would explain the weaker capture effect for the color cue condition, as in this case no match was present between the features of the cue and the target. As top-down modulation would have been precluded, there could be only brief attentional engagement at the color singleton's site. (c) According to previous work (Folk \& Remington, 1998, 2008; Lamy et al., 2006b), the ACS would be narrowly tuned to monitor the specific features that define targethood, 
so capture by these features would be stronger than capture by a feature for which the ACS was not specifically tuned. Experiment 2 was designed to examine whether capture by an irrelevant color cue could be enhanced when the ACS was tuned to detect its specific value.

\section{Experiment 2}

Some data have suggested that, when the diagnostic target feature is predictable, the ACS would be narrowly tuned to monitor this feature (Folk \& Remington, 1998, 2008; Lamy et al., 2006b). In addition, Folk and Anderson (2010) demonstrated that the ACS can be narrowly tuned to more than one value on a single dimension. The question asked in Experiment 2 was whether this narrow tuning could be extended to two different dimensions. In Experiment 1, a larger capture effect was observed for the onset cue as compared to the color cue. There, color was not the target's defining feature. Experiment 2 was, in essence, a replication of Experiment 1, except for one crucial change: Color was made a diagnostic target feature. As in Experiment 1, the target location was marked by a dynamic discontinuity (abrupt appearance of a second ring) and a shape singleton (two concentric rings around the target), but in Experiment 2 it was also marked by a color singleton (the second ring had a unique color). As the irrelevant color cue would, like the onset cue, benefit from the top-down modulation afforded by a match between the features of cue and target, there should be comparable cuing effects.

\section{Method}

Observers A group of 15 observers was recruited from the same undergraduate pool.

Procedure The design and execution of the experiment were identical to those aspects of Experiment 1. Two versions were conducted that differed in the color used as one of the target's defining features. Version 1 is described first. In the color singleton cue condition, one set of spots turned blue, and the other three sets of spots were red (see the second frame of Fig. 3). In the onset cue condition, a set of four purple spots appeared abruptly around one of the filled circles, and simultaneously, the spots surrounding two unfilled circles also turned purple (CIE[Yxy]: 29.8, .31, .22). When the search letters were revealed, the existing circles turned green. In addition, a blue ring appeared abruptly, surrounding the circle that enclosed the target letter (see the third frame of Fig. 3). The color of this additional ring matched the color of the irrelevant color singleton described earlier. Thus, three unique and correlated features appeared at the target letter's site: a dynamic (onset) transient, a shape (two concentric circles), and a color (blue) singleton. In

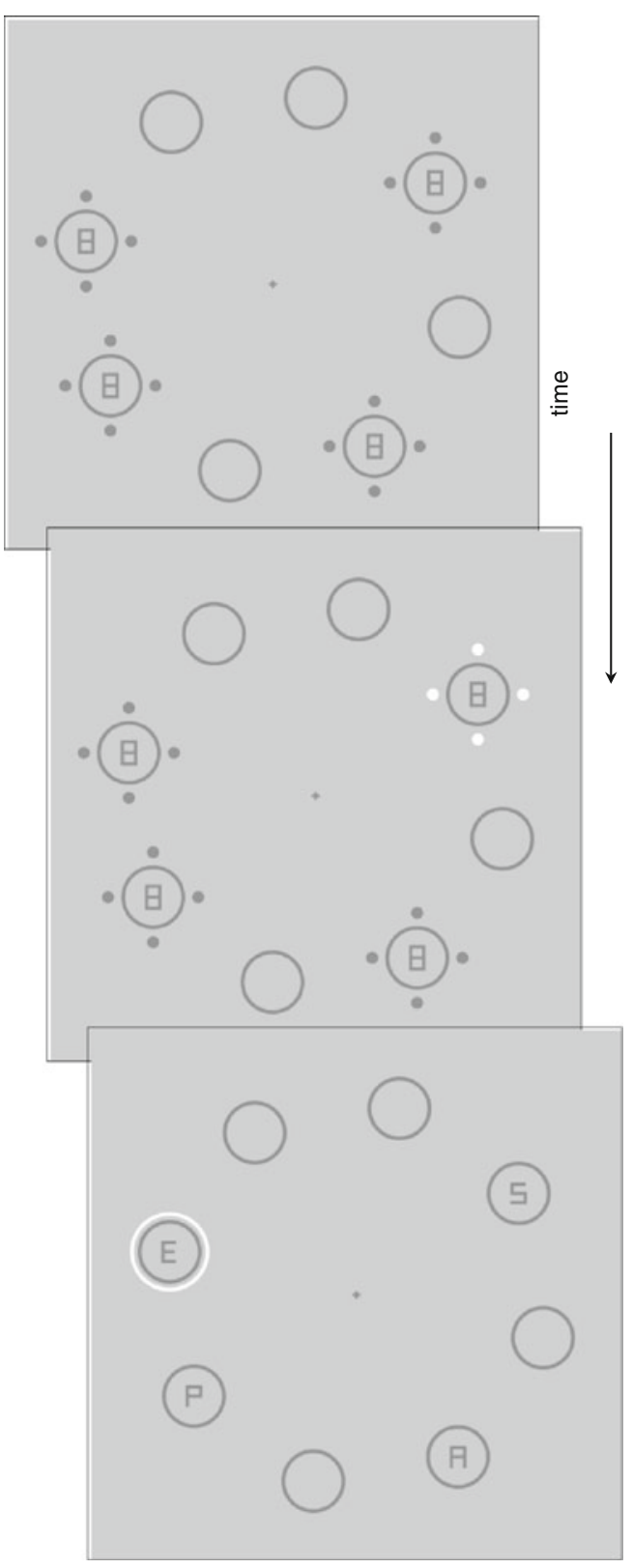

Fig. 3 Experiment 2, irrelevant-color-singleton cue condition: Frames 1-3, from top to bottom. In Frame 1, the display was ramped up. In Frame 2, the spots surrounding the filled circles changed from gray to a color, with three sets of spots turning one color, and the remaining set turning a different color (white, in the figure). In Frame 3, the search array was presented. An additional ring appeared abruptly around the circle enclosing the target letter. This additional ring had a different color from the rest, making it a color singleton (white, in the figure)

Version 2, the additional ring that appeared around the target letter was red, as was the irrelevant color singleton, to match the color of the additional target ring. In contrast, the nonsingletons were blue. In all other respects, the two versions were identical. The instructions to the observers were identical to those of Experiment 1: The subjects were briefed to look for the two concentric rings and to identify the letter enclosed within. 
Results

The latency and error data of the validity conditions, as a function of the type of irrelevant cue, are presented in Fig. 4.

Analysis of the accuracy data showed no effects whatsoever, suggesting that a speed-accuracy trade-off was not an issue with these data. ${ }^{2}$ The mean latencies were longer for the less accurate conditions.

For the main analysis of the latency data, a 2 (version) $\times 2$ (cue type) $\times 3$ (cue validity) ANOVA revealed only a cue validity effect, $F(2,26)=28.826, p<.001, \eta_{\mathrm{p}}^{2}=.69$. The version variable was not significant, $F<1$, nor did it interact reliably with any of the other variables, $F \mathrm{~s}<1$. The cue type and Cue Type $\times$ Cue Validity effects were not significant, $F<$ $1, F(2,26)=1.950, p<.2, \eta_{\mathrm{p}}{ }^{2}=.13$. The pattern in Experiment 1 was different from these results, which a cross-experiment analysis confirmed. ${ }^{3}$

\section{Discussion}

In contrast to Experiment 1, comparable capture effects were found for the color and onset cues in Experiment 2. Singleton detection was a viable strategy in both experiments, and had this strategy been adopted, comparable cuing effects for the two experiments would have been found. Experiment 2's results are therefore consistent with data that have shown the ACS can tune narrowly to a specific target feature.

Obtaining capture effects of comparable magnitude for the color and onset cue conditions showed that the onset cue, at least as it was manipulated in this experiment, was not inherently more salient than the color cue. The results implicated top-down modulation as an important factor in explaining capture magnitude. This point is well-illustrated in the color singleton conditions in Experiments 1 and 2. The match between the colors of the cue and target in Experiment 2 would have induced top-down modulation. This would account for the enhanced capture effect. In Experiment 1, there was no target-cue match; thus, the capture effect was smaller, but this smaller effect was

\footnotetext{
${ }^{2}$ A 2 (version) $\times 2$ (cue type) $\times 3$ (cue validity) ANOVA revealed no effects whatsoever: $F<1 ; F(1,14)=1.519, p<.3$; and $F(2,28)=$ $2.498, p<.2$, for the version, cue type, and cue validity variables, respectively. The interaction effects were not reliable, the largest being the three-factor interaction, $F(2,26)=2.423, p<.2$.

${ }^{3}$ The data were collapsed across the version variable for the crossexperiment analysis. A 2 (experiment: 1 vs. 2 ) $\times 2$ (cue type) $\times 3$ (cue validity) ANOVA revealed no cue type effect, $F<1$, nor a Cue Type $\times$ Experiment interaction, $F<1$. An overall cue validity effect did emerge, $F(2,56)=63.204, p<.001$, but no Cue Validity $\times$ Experiment interaction, $F<1$. Furthermore, there was an overall Cue Type $\times$ Cue Validity interaction, $F(2,56)=6.756, p<.005$, and crucially, the threefactor interaction effect was also reliable, $F(2,56)$ $=6.150, p<.005$.
}

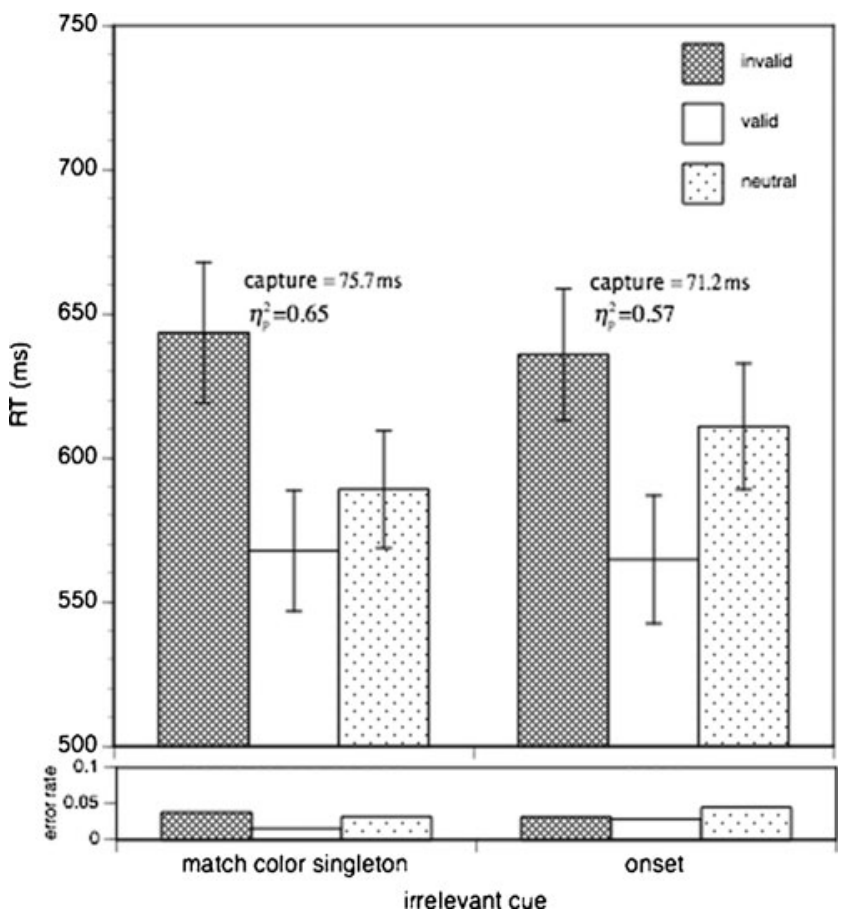

Fig. 4 Experiment 2: Mean RTs and error rates of the valid, invalid, and neutral cue conditions as a function of type of irrelevant cue. The magnitude of the capture effect is computed by taking the difference between the invalid and valid cue conditions, and capture effect size is also reported. Error bars $=1 S E$

nevertheless reliable. Experiment 3 was designed to examine this smaller capture effect, to determine whether it was due to the singleton's inherent saliency or constituted a muted form of top-down modulation.

The assumption, so far, has been that all of the features diagnostic of targethood are programmed into the ACS. An alternative hypothesis would be that, at the start of each trial, the system randomly picks just one of these features and programs the ACS accordingly. This hypothesis would predict that, averaged across all trials, there would still be a capture effect. However, the hypothesis also predicts an increased probability of a match between the cue and the randomly selected target feature when the target has fewer diagnostic features. Therefore, the capture magnitude in this case should also increase. The onset condition in Experiments 1 and 2 had allowed a test of this hypothesis: Two target features were diagnostic in Experiment 1, but three in Experiment 2. According to this alternative hypothesis, capture magnitudes ought to have been larger in Experiment 1. The results of a cross-experiment ANOVA contradicted this prediction of the hypothesis. ${ }^{4}$ Furthermore,

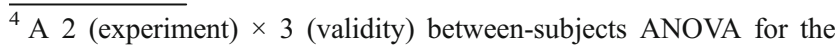
onset cue condition revealed only a validity effect, $F(2,56)=47.449, p$ $<.001, \eta_{\mathrm{p}}{ }^{2}=.63$. The experiment effect and, crucially, the interaction were not significant, $F(1,28)=1.429, p>.2, \eta_{\mathrm{p}}{ }^{2}=.05$, and $F(2,56)=$ $1.417, p>.2, \eta_{\mathrm{p}}{ }^{2}=.05$, respectively.
} 
in an experiment in which the onset cue was the only diagnostic feature, Chua (submitted) reported an $\sim 100$-ms capture effect. When the system was set to monitor onset transients, the onset cue's capture effect was fairly consistent across experiments. Whether the onset transient was the only diagnostic cue, or one of several, did not seem to matter.

Experiment 2 demonstrated that top-down modulation enhanced attentional capture. Experiment 3 was designed to explain how a color singleton cue succeeded in capturing attention when it shared no features with the target. Lamy et al. (2006b) argued that the ACS could be narrowly tuned to monitor a specific target feature but also, simultaneously, broadly tuned to detect singletons. Their conclusion was based on data gathered using the irrelevant-singleton paradigm. Experiment $3 \mathrm{a}$ was designed to test the generality of their argument with a spatial-cuing paradigm, and Experiment $3 \mathrm{~b}$ to examine the saliency explanation.

\section{Experiment 3}

In Experiment 1, the irrelevant color singleton cue captured attention although it did not share any of the target's diagnostic features. As the target location was demarcated by both a static and a dynamic discontinuity, the system could have been set broadly to monitor singletons. This would explain why a nonmatching color singleton cue succeeded in capturing attention. An alternative explanation assumes that a salient cue, such as a color singleton, automatically captures attention in a stimulus-driven fashion. According to Theeuwes's (2010) disengagement hypothesis, this initial bottom-up capture is likely to be brief unless it is followed by top-down modulation, which will only occur if the salient cue shares the target's features. The contrasting patterns in the results of Experiments 1 and 2 are consistent with this hypothesis.

Experiment 3a was designed to examine the Lamy et al. (2006b) argument that the ACS can be narrowly tuned to specific target features and, simultaneously, broadly tuned to detect singletons. Some data in the literature appear to contradict this view of a flexibly tuned ACS. For example, Folk and Remington $(1998,2008)$ reported that when the target was a red singleton, an irrelevant green singleton cue failed to capture attention, suggesting a narrowly tuned ACS. In Folk and Remington's work, only one (color) dimension was relevant, which might have precluded observing both narrow and broad tuning at the same time. In Experiment $3 \mathrm{a}$, the target location was marked by unique values on three dimensions (cf. Lamy et al., 2006b). The question was whether simultaneous narrow and broad tuning would be observed in this context.
Experiment 3a

\section{Method}

Observers Another group of 15 students were recruited as subjects.

Procedure The design and conduct of this experiment were identical to those of Experiment 2. When the search letters were revealed, the existing circles turned yellow (CIE[Yxy]: $29.9, .50, .42$ ). An additional blue ring appeared abruptly around the target letter. Thus, the target location was marked by (a) a dynamic discontinuity and (b) a static discontinuity that had a unique shape (two concentric circles among single circles in nontarget locations) and color (a blue ring among yellow rings). Two types of irrelevant color singleton cues were compared: one set of blue spots among three sets of red spots, with the unique blue spots matching the color of the additional ring around the target, and one set of green spots (also among red spots), in which there was no match to the colors presented in the search frame.

\section{Results and discussion}

The latency and error data of the validity conditions as a function of the type of irrelevant cue are presented in Fig. 5. A preliminary analysis of the error results showed that a speed-accuracy trade-off was not an issue with these data. ${ }^{5}$ For the main analysis, the latency data were subjected to a 2 (cue type: match vs. mismatch cue) $\times 3$ (cue validity) ANOVA. No overall main effect of cue type emerged, $F<$ 1 . The cue validity variable was significant, however, with mean RT being shortest for the valid, and longest for the invalid, cue conditions, $F(2,26)=54.372, p<.001, \eta_{\mathrm{p}}{ }^{2}=$ .81. Crucially, an interaction was also found, with the cue validity effect $(102 \mathrm{~ms})$ being larger for the cue match condition, $F(2,26)=17.497, p<.001, \eta_{\mathrm{p}}{ }^{2}=.57$. Although the effect for the cue mismatch condition (31 ms) was smaller, it was nevertheless reliable, $F(2,26)=9.086, p<.005, \eta_{\mathrm{p}}{ }^{2}=.41$.

The pattern in the results here replicated those of the color singleton conditions in Experiments 1 and 2. In Experiment 3a, a larger color capture effect appeared for the irrelevant blue spots (which matched the unique target color feature) than the nonmatch green spots. Capture by the color singleton in the match condition was predicted by the contingent-orienting hypothesis, but the more interesting finding was the smaller, but nonetheless reliable, capture effect by the nonmatch singleton. First, if the ACS had been narrowly tuned only to the target color, there would not be

\footnotetext{
$\overline{\left.{ }^{5} \text { A } 2 \text { (cue type }\right)} \times 3$ (cue validity) ANOVA on the error data revealed no significant effects, $F \mathrm{~S}<1$, for the main effects, and $F(2,38)=2.184$, $p>.1$, for the interaction effect.
} 


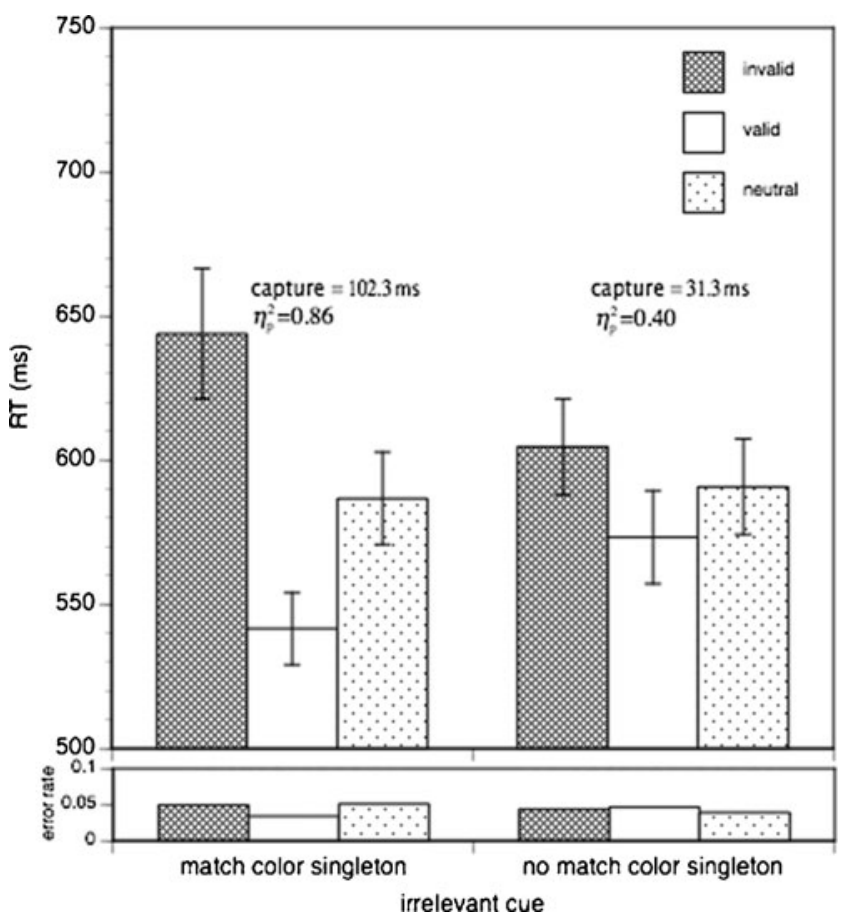

Fig. 5 Experiment 3a: Mean RTs and error rates of the valid, invalid, and neutral cue conditions as a function of type of irrelevant cue. The magnitude of the capture effect is computed by taking the difference between the invalid and valid cue conditions, and capture effect size is also reported. Error bars $=1 S E$

evidence of spatial cuing for the nonmatch condition (e.g., Folk \& Remington, 1998, 2008). Second, the results contradicted the hypothesis of a default singleton-detection search mode (Bacon \& Egeth, 1994), which would have predicted comparable capture effects for the match and nonmatch conditions.

As was noted earlier, Folk and Remington $(1998,2008)$ used a single relevant dimension, which might have precluded demonstrating broad and narrow tuning on that one dimension. In Experiment 3a, the target's multiple unique features could have enhanced its status as a singleton. Topdown modulation of the narrowly tuned color dimension may have been foreclosed for the nonmatch color singleton condition. But, as the system was also tuned to detect singletons, another source of top-down modulation was available. The latter appears to be a weaker form of top-down modulation than the modulation that was obtained when the cue matched the target.

\section{Experiment $3 b$}

The results of Experiment 3a (and Exp. 1) were consistent with an account that assumes that singleton detection was included as a top-down search strategy. But capture by the nonmatch color singleton cue could just as well be attributed to its saliency (e.g., Theeuwes, 2010). According to the saliency account, a salient stimulus automatically captures attention in a stimulus-driven fashion, but if subsequent topdown modulation is denied, the overall capture effect would be weak. Experiment $3 \mathrm{~b}$ was designed to directly test the saliency account, as the search environment was configured to thwart a singleton-detection strategy. Consider now the nonmatch color singleton cue in Experiment 3a. If the attentional orienting to this cue were due to singleton detection, foreclosing this strategy would also stymie capture by this cue. On the other hand, the cue's saliency ought not to be altered by any change in the search environment.

\section{Method}

Observers An additional 15 observers were recruited from the same subject pool under the same motivational conditions.

Procedure The crucial difference between Experiments 3a and $3 \mathrm{~b}$ was that, in the latter experiment, the target location was not marked by a unique discontinuity. The frame sequence is depicted in Fig. 6. When the search array was presented, all of the circles turned green (third frame). At the target location, an additional, larger green circle appeared abruptly around the green circle that enclosed the target letter. Furthermore, two additional green circles also appeared, each surrounding an empty green circle. Thus, the target location was not marked by a unique shape discontinuity, nor by a unique onset transient. As in Experiment 1, color was not a diagnostic target feature.

The design and conduct of this experiment followed those of the previous experiments. Two main variables were tested: (a) type of irrelevant color cue and (b) cue validity. There were two types of irrelevant color cue. In the color singleton condition, one set of spots turned blue, and the other three sets turned red (not shown in Fig. 6). In the nonsingleton condition, the spots in the valid or the invalid locations turned blue, matching the color singleton cue in the singleton condition (second frame, at $\sim 1$ o'clock position, Fig. 6). One set each of the remaining three sets of spots turned red, purple, or yellow (see Fig. 6). As capture was not expected for the nonsingleton condition, it constituted the baseline on which to evaluate the singleton condition.

\section{Results}

The latency and error data of the validity conditions as a function of the type of irrelevant cue are presented in Fig. 7. The preliminary analysis of the error data revealed no interesting trends. ${ }^{6}$ Speed-accuracy trade-off was not an issue with these data.

\footnotetext{
$\overline{{ }^{6} \text { A } 2 \text { (cue type) }} \times 3$ (cue validity) ANOVA using error rate as the dependent variable revealed no significant effects, all $F_{\mathrm{s}}<1$.
} 


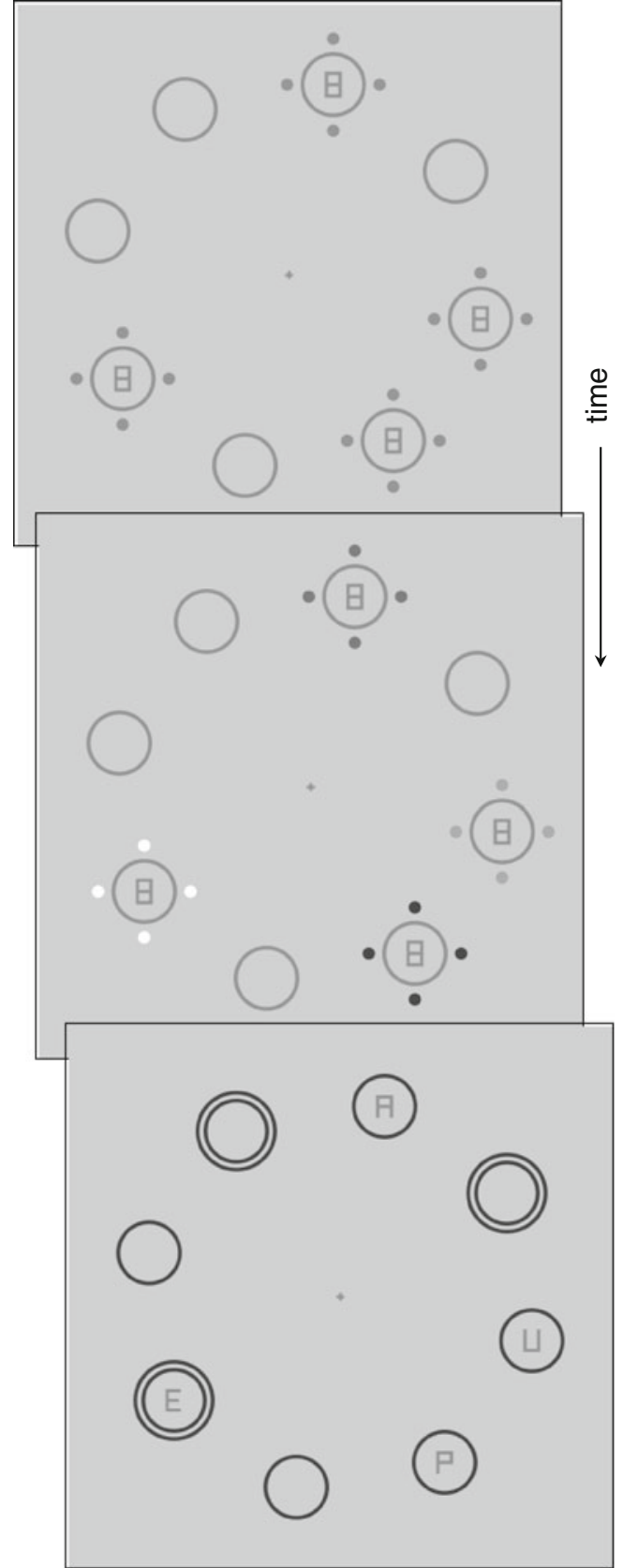

Fig. 6 Experiment 3b, irrelevant-color-singleton cue condition: Frames 1-3 (top to bottom). In Frame 1, the display was ramped up. In Frame 2, the spots surrounding the filled circles changed color, each set of spots changing to a color different from the rest (black, white, and different shades of gray in the figure) In Frame 3, the search array was presented. An additional ring appeared abruptly around the circle enclosing the target letter, and other additional rings appeared around two unfilled circles

The search environment was designed to make singleton detection a nonviable strategy. Before analyzing the latency data, it is important to ask whether we succeeded in this goal. The data suggest that the observers' mean search latencies were reliably longer in Experiment $3 \mathrm{~b}$ (690 vs. $590 \mathrm{~ms}$ for Exp. 3a). An independent-samples

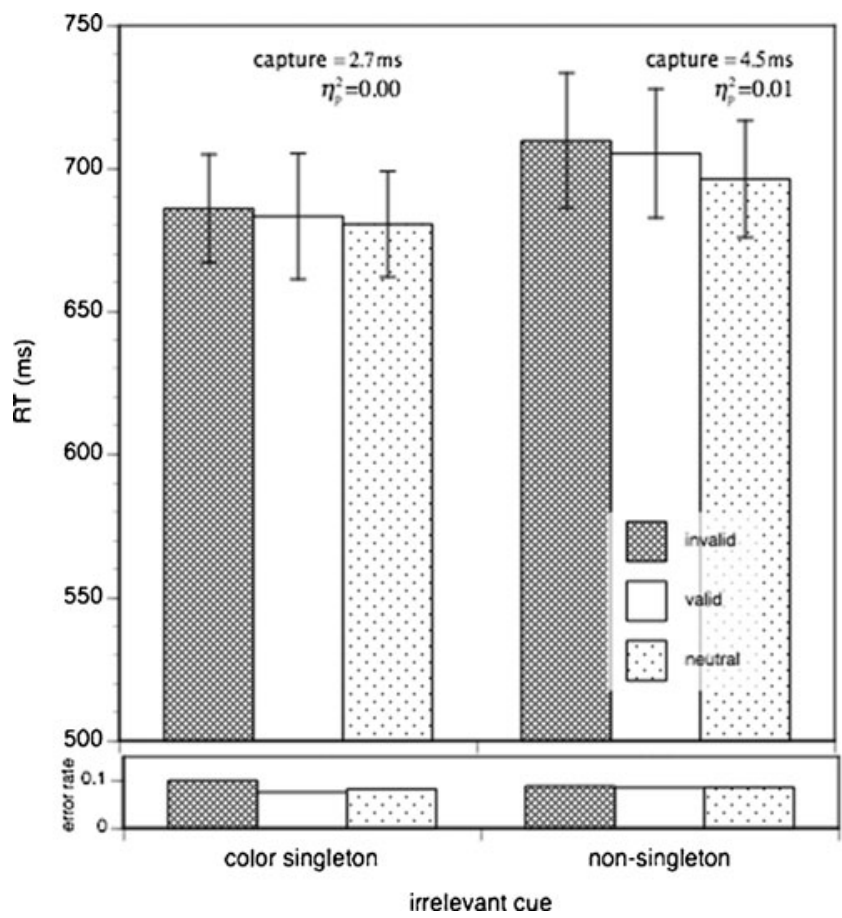

Fig. 7 Experiment 3b: Mean RTs and error rates of the valid, invalid, and neutral cue conditions as a function of type of irrelevant cue. The magnitude of the capture effect is computed by taking the difference between the invalid and valid cue conditions, and capture effect size is also reported. Error bars $=1 S E$

test of the overall search times in Experiments $3 a$ and $3 b$ (i.e., collapsed across the cue type and cue validity variables) showed that search was harder in Experiment 3b, $F(1,28)=18.117, p<.001 .^{7}$

For the main analysis, a 2 (cue type: blue singleton vs. nonsingleton) $\times 3$ (cue validity) ANOVA was conducted on the latency data. No significant effects were found, all $F_{\mathrm{S}}<$ 1. As expected, the nonsingleton cue failed to capture attention. The failure of the irrelevant singleton cue to capture attention (capture effect $=3 \mathrm{~ms}$ ) stands in marked contrast to the finding that the same cue succeeded in Experiment $3 \mathrm{a}$ (capture effect $=31 \mathrm{~ms})^{8}$

\section{Discussion}

Singleton detection was a viable strategy in Experiment 3a, but not in Experiment 3b. The salience of the irrelevant

\footnotetext{
${ }^{7}$ The same analysis, comparing Experiments 1 (in which the additional ring had the same color as the distractors) and $3 \mathrm{~b}$, yielded consistent results, $F(1,28)=6.871, p<.02$.

${ }^{8}$ A 2 (experiment) $\times 3$ (cue validity) ANOVA comparing the latency data of the nonmatching singleton cue revealed an overall experiment effect, $F(1,28)=13.424, p<.001$. When the target could not be localized via a discontinuity (Exp. 3b), latencies were significantly longer. No overall cue validity effect emerged, $F(2,56)=1.399, p>$ .2 , but crucially, the Experiment $\times$ Cue Validity interaction effect was reliable, $F(2,56)=4.496, p<.02$.
} 
nonmatching color singleton cue was the same in these two experiments. Thus, if attentional orienting were due to the cue's salience alone, the capture effects ought to have been comparable across the two experiments. The contrast between the results rules out the saliency hypothesis.

The results here recall a similar experiment reported by Lamy, Leber, and Egeth (2004), in which their observers were also compelled to adopt a feature-search strategy. Lamy et al. (2004) compared a color singleton with a nonsingleton cue. The latter cue was, by definition, not salient. Although both cues matched the defining target color, the saliency account would predict capture only by the singleton cue. Lamy et al. (2004) reported comparable capture effects for the two cues, implying that bottom-up mechanisms did not play a substantive role in capture.

Experiment $3 \mathrm{~b}$ may be considered complementary to Lamy et al. (2004). According to the saliency account, the search environment should not affect bottom-up attentional orienting. The results, consistent with those of the earlier study, showed that the color singleton's saliency was not a crucial factor. In addition, Lamy et al. (2004) also reported an inhibition effect in their nonmatching singleton cue condition. For the comparable condition in Experiment 3b, a null result was found. The difference between the results of the two experiments could lie in how the diagnostic target feature was manipulated in the experiments. In Lamy et al. (2004), the defining target feature and the nonmatching singleton cue were values on the same (color) dimension. In Experiment 3b, the target had several diagnostic features in different dimensions.

\section{Experiment 4}

Folk and Anderson (2010) reported that, in some search contexts, the ACS can be narrowly tuned to more than one value on a single dimension. The results reported in Experiments 13 , which showed narrow tuning to more than one dimension, extend the Folk and Anderson results. The question posed in Experiment 4 was whether this narrow tuning pertained to all of the target's features, or only to those features that were diagnostic of targethood. More specifically, the question was whether a feature that the target shares with the nontargets also gets prioritized? The focus of interest was an irrelevant color singleton cue that bore the target's nondiagnostic color feature. If the complete inventory of the target's features was programmed into the ACS, this color cue should receive topdown attentional modulation.

Method

Observers A group of 16 subjects took part in this experiment. They were recruited from the same pool, and under the same motivational conditions, as in the previous experiments.

Procedure Two types of irrelevant color singleton cues were compared: (a) spots that did not match the circles' color (nonmatch condition) and (b) spots in which there was a match (match condition). In the match condition, the spots' color also matched the color of the nontarget rings. Two versions were conducted, which differed in the color of the rings presented in the search frame. In Version 1, the rings were green, and the irrelevant color singleton spots were either blue (nonmatch condition) or green (match condition). The nonsingleton spots were red. In Version 2, the rings were red, and the singleton spots were green and red, respectively, for the nonmatch and match conditions. The nonsingleton spots here were blue. An additional ring appeared around the target letter's existing enclosure when the search array appeared. All of the rings had the same color.

Results and discussion

The latency and error data of the validity conditions, as a function of the type of irrelevant cue, are presented in Fig. 8. The data showed a positive relationship between latency and accuracy, suggesting that speed-accuracy trade-off did not pose a problem. A formal analysis of the accuracy data confirmed this. ${ }^{9}$

For the main analysis, the latency data were analyzed in a 2 (version) $\times 2$ (cue type) $\times 3$ (cue validity) mixed-factor ANOVA, with Version treated as a between-subjects factor. The two versions were comparable, $F<1$. No significant interaction emerged between versions and the other two variables. There was an overall cue validity effect, $F(2$, 28) $=20.438, p<.001, \eta_{\mathrm{p}}{ }^{2}=.59$, but neither the cue type nor the interaction effect was significant, $F_{\mathbf{S}}<1$.

As the target location was marked by unique spatial and dynamic discontinuities, it would be expected, from the results of Experiments 1 and 3a, that singleton detection would be an aspect of the attentional system's top-down strategy. Therefore, attentional orienting should occur toward the nonmatch color singleton cue. The results supported this prediction. A consistent finding in Experiments 1-3a was that the capture magnitude was larger when the singleton cue's unique feature matched one of the target's diagnostic features. The crucial question was whether the same result would be seen in this experiment for the match condition. If the nondiagnostic color

\footnotetext{
$\overline{9}$ A 2 (cue type) $\times 3$ (cue validity) ANOVA revealed only a single cue validity effect, $F(2,30)=4.953, p<.05$. All of the other effects were not reliable, $F \mathrm{~s}<1$. There was an inverse relationship between accuracy and latency, suggesting that speed-accuracy trade-off was not an issue.
} 


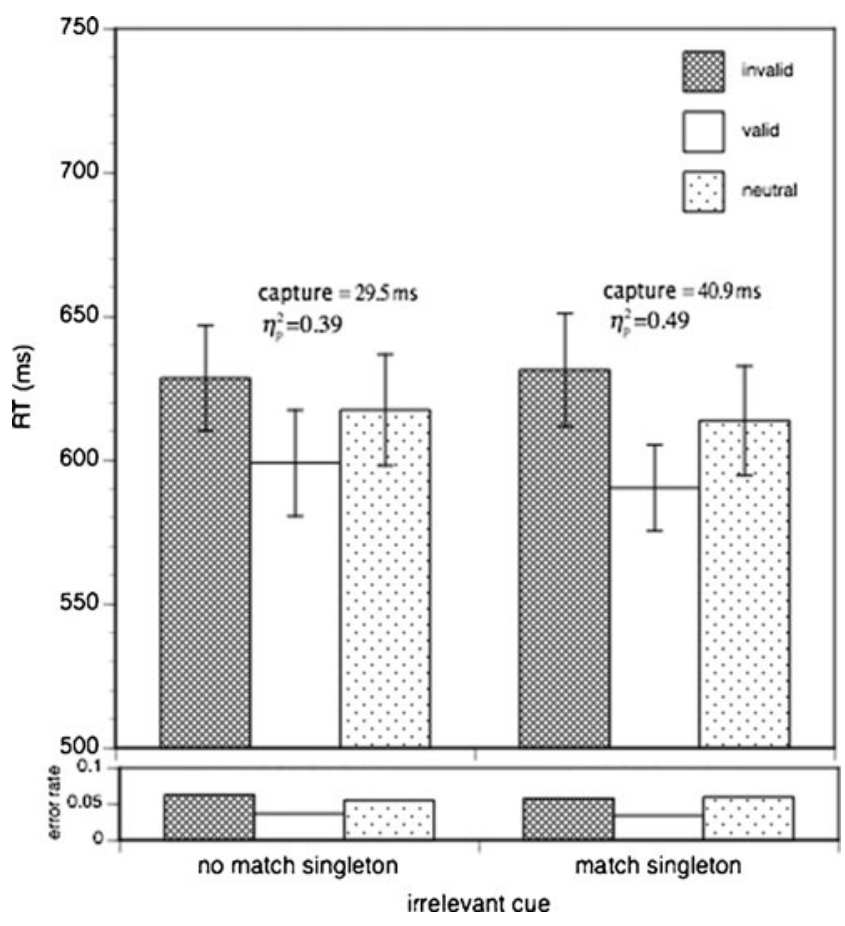

Fig. 8 Experiment 4: Mean RTs and error rates of the valid, invalid, and neutral cue conditions as a function of type of irrelevant cue. The magnitude of the capture effect is computed by taking the difference between the invalid and valid cue conditions, and capture effect size is also reported. Error bars $=1 S E$

of the target had been programmed into the ACS, there should be a stronger attentional orienting effect in the match than in the nonmatch condition. The results of Experiment 4 showed that the spatial-cuing effect was larger for the match (41 ms, $\left.\eta_{\mathrm{p}}{ }^{2}=.49\right)$ than for the nonmatch $\left(29 \mathrm{~ms}, \eta_{\mathrm{p}}{ }^{2}=.39\right)$, condition. The difference, however, was not statistically reliable, suggesting that the target's nondiagnostic feature had been excluded from the ACS. This pattern contrasted with the results of Experiment 3a, as was verified by a cross-experiment analysis. ${ }^{10}$ The implication is that features of the target that were not diagnostic of targethood were not prioritized.

\section{General discussion}

Consider a search task in which a target, possessing several unique features, is embedded in a field of homogeneous distractors. How might the system guide the attentional

\footnotetext{
${ }^{10}$ A 2 (experiment: 3 a vs. 4 ) $\times 2$ (cue type: match vs. nonmatch color singleton) $\times 3$ (cue validity) mixed ANOVA, treating Experiment as a between-subjects factor, revealed no experiment, cue type, or Experiment $\times$ Cue Type effects, $F \mathrm{~s}<1$. An overall cue validity effect was found, $F(2,58)=75.013, p<.001, \eta_{\mathrm{p}}{ }^{2}=.72$, as well as an Experiment $\times$ Cue Validity interaction, $F(2,58)=7.435, p<.01, \eta_{\mathrm{p}}{ }^{2}$ $=.20$. Furthermore, a Cue Type $\times$ Cue Validity interaction emerged, $F$ $(2,58)=11.876, p<.01, \eta_{\mathrm{p}}^{2}=.29$. Finally, a three-factor interaction was also apparent, $F(2,58)=6.224, p<.005, \eta_{\mathrm{p}}{ }^{2}=.18$.
}

spotlight swiftly and efficiently to the target? As detecting any of the target's features would lead, unerringly, to the target, it would seem that a strategy of monitoring all of the target's features would be cognitively inefficient. Indeed, instead of tuning the detectors to any specific target feature, a strategy of detecting the local discontinuity would work just as well. Two interesting findings emerged from this set of experiments. First, instead of prioritizing the single most salient cue or relying only on a bottom-up saliency detection strategy, the attentional system appeared to be configured to monitor the entire set of unique target features. Second, to accomplish this, observers' ACSs were flexibly tuned to monitor simultaneously a range of discontinuities, including static and dynamic discontinuities.

The experiments in this article were based on the spatialcuing paradigm, in which an irrelevant cue was presented before the search objects appeared (Folk et al., 1992). The attentional system's prioritization scheme was inferred from the pattern of attentional orienting to the irrelevant cue. According to the contingent-orienting hypothesis (Folk et al., 1992, 1994), prioritization of a target feature should lead to involuntary orienting to a cue that bears this feature. The cue's capture success was assessed by comparing search latencies for trials on which the target's location was coincident with the cue (the valid condition) and on which these locations were different (the invalid condition). If there was no orienting to the cue, the RTs for these conditions should be comparable. Otherwise, a longer RT for the invalid condition should be observed.

The findings of this set of experiments may be summarized as follows:

1. The ACS was tuned narrowly to monitor a specific feature, and simultaneously broadly to detect singletons (Exps. 1 and 3);

2. The ACS was programmed to monitor, simultaneously, spatial and dynamic discontinuities (Exp. 2);

3. Features that were not diagnostic of the target did not appear to be programmed in the ACS (Exp. 4).

Simultaneous narrow and broad tuning

When the distractors in the search environment are heterogeneous, localizing the target requires the search mechanisms to be narrowly tuned to the specific target features (e.g., Lamy et al., 2004). This finding raises the question of whether the same narrow tuning would also be used when a singleton-detection strategy was feasible. Consider, for example, Theeuwes's (1992) study, in which he showed attentional capture by an irrelevant (color) singleton when the diagnostic target feature was defined according to a different (shape) dimension. As the objects' colors were irrelevant, Theeuwes assumed that his observers monitored only the 
unique shape that enclosed the target. According to this assumption, attentional capture by the color singleton would constitute evidence of stimulus-driven attentional orienting.

The complication in Theeuwes's (1992) design was that the shapes enclosing the target and the irrelevant distractor were both singletons (shape and color, respectively). Bacon and Egeth (1994) argued that this search environment allowed a singleton-detection strategy to be employed. If search had been guided by this strategy, one should not count capture by the irrelevant color distractor as an instance of bottom-up, stimulus-driven capture. Bacon and Egeth showed that the irrelevant color cue only succeeded in capturing attention when singleton detection was a viable strategy - that is, when the target was also a singleton. When this strategy was foreclosed, the same color cue failed to capture attention. Bacon and Egeth's contention was that, so long as the target is a singleton, the attentional system would default to a singleton-detection strategy.

Lamy et al. (2006b) noted that the Bacon and Egeth (1994) claim appears inconsistent with studies demonstrating that knowledge of the specific target feature(s) guided search for a singleton target (e.g., Bravo \& Nakayama, 1992; Maljkovic \& Nakayama, 1994). Thus, contrary to Bacon and Egeth's assumption, the ACS could be narrowly tuned to the singleton target's features. The important contribution of Lamy, Carmel, et al.'s. results was that they suggested a more flexible attentional system than is allowed by the standard assumption of a singleton-detection versus feature-search dichotomy. Lamy, Carmel, et al. compared a condition in which the target singleton remained fixed (therefore, predictable) with a condition in which the target's form varied unpredictably across trials. They reported a speed advantage for the fixed-target condition, thereby implicating a narrowly tuned ACS. But, while distraction from the irrelevant singleton diminished in the fixed-target condition, the effect remained significant. This last result was essentially what Theeuwes (1992) had found. Lamy, Carmel, et al.'s interpretation, which departed from those of Theeuwes and of Bacon and Egeth, was that the ACS could simultaneously adopt a feature-search and a singleton-detection strategy. The important theoretical point here was that the two strategies should not be seen as mutually exclusive.

For the experiments reported in this article, the target (a) was a singleton, which (b) had several unique features, all of which (c) remained constant throughout. Extending from recent findings, the prediction was that the observers' ACSs would be narrowly tuned to the target's features. Of particular interest was the fate of an irrelevant singleton cue that matched none of the target's several diagnostic features (Exps. 1, 3, and 4). A strong version of the contingentorienting hypothesis would predict that the nonmatching singleton cue should fail to capture attention (e.g., Folk \&
Remington, 1998, 2008). According to Bacon and Egeth's (1994) view, no difference should emerge between the matching and nonmatching singleton conditions. The results showed that while the nonmatching color singleton cue captured attention, the magnitude of its capture effect was smaller than when the cue matched a target feature.

There are two ways to explain why a nonmatching singleton cue could still succeed in capturing attention. First, it could be considered an instance of bottom-up, stimulusdriven capture (e.g., Theeuwes, 2010). Alternatively, following Lamy et al. (2006b) lead, the capture effect could be the consequence of an ACS programmed to detect specific target features, and also singletons.

These two alternative accounts were tested in Experiment 3. Experiment $3 \mathrm{a}$ replicated and extended the findings of Experiments 1 and 2. In Experiment 3b, however, singleton detection was precluded as a viable strategy. Changing the search environment should have no implications for the color singleton's saliency per se, so if attentional orienting was driven by bottom-up saliency effects, capture should still be obtained for the color singleton, as compared to the nonsingleton, condition. The results contradicted the predictions of the saliency hypothesis.

An issue that Theeuwes (2010) raised was whether the spatial-cuing paradigm constitutes an optimal platform to evaluate the saliency account. According to him, the duration of preattentive capture is very brief. When the cuetarget stimulus onset asynchrony is long (as in these experiments), attention in the nonmatch condition would almost certainly have disengaged when the search letters were revealed. Aspects of the data appear consistent with Theeuwes (2010). For example, capture magnitude was smaller in the nonmatch than in the match condition, consistent with the notion that top-down attentional modulation augments the effect due to an initial bottom-up capture. As these experiments were not designed to test the disengagement hypothesis, we remain agnostic and await data that speak directly to this hypothesis.

\section{Tuning to static and dynamic discontinuities}

In considering the range of features that might be registered with the ACS, Folk et al. (1992; Folk et al., 1994) distinguished between static and dynamic discontinuities as two broad categories. Folk et al. (1994) speculated that these two types of discontinuities could be monitored by separate pathways. In their initial version of the contingentorienting hypothesis, they assumed that the ACS would be broadly tuned, which implied that the system can only be programmed to respond to one or the other discontinuity, but not to both simultaneously.

Recent findings suggest that the tuning function is narrower than their earlier conception (e.g., Folk \& Remington, 
1998, 2008), which allows for the possibility that the attentional system may be set to monitor, simultaneously, static and dynamic discontinuities. Experiments 1 and 2 focused on this issue. In Experiment 1, the target was both a shape and an onset singleton. Comparable capture effects were observed for the onset conditions in the two experiments, as confirmed by a cross-experiment analysis. ${ }^{11}$ As the target location was marked by a unique onset transient, these results imply that the attentional system was set to monitor a dynamic discontinuity. ${ }^{12}$

The irrelevant-color-singleton condition is of particular interest. In Experiment 1, there was no match between the cue's unique feature (color) and the target's unique features (shape and onset). In Experiment 2, color was added as another of the target's unique features, thereby allowing a match between cue and target. If the system has no capacity to monitor simultaneously static and dynamic discontinuities, adding another unique static feature to the target (in Exp. 2) should not, materially, change the capture dynamics. That is, had the color feature not been prioritized, the system would, in a sense, still be blind to the match between the cue and the target on this dimension. Experiments 1 and 2 revealed a different pattern of spatial cuing to the irrelevant color singleton cue. In Experiment 2, spatial cuing to the irrelevant color cue was comparable to the onset cue, suggesting that the system had been tuned to the specific target color.

\section{Tuning to multiple cues}

Search will be efficient so long as the target is a singleton. One implication of the results presented in this article is that, even in an efficient search environment, the attentional system appeared to be set to monitor the full set of unique target features. In some contexts, this might constitute an optimal strategy. Consider the case in which the target is

\footnotetext{
${ }^{11}$ A 2 (Exp. 1 vs. 2$) \times 3$ (cue validity) ANOVA conducted on the mean RTs of the onset condition revealed only a cue validity effect, $F(2,56)$ $=47.449, p<.001, \eta_{\mathrm{p}}{ }^{2}=.63$. The experiment effect and the Experiment $\times$ Cue Validity interaction were not reliable, $F(1,28)=$ $1.429, p>.2, \eta_{\mathrm{p}}{ }^{2}=.05$, and $F(2,56)=1.417, p>.2, \eta_{\mathrm{p}}{ }^{2}=.05$, respectively.

${ }^{12}$ Could capture by the onset cue be attributed to stimulus-driven orienting? As an onset transient was one of the diagnostic target features, it would be more parsimonious to assume that the onset capture was due to top-down modulations. Furthermore, it has been shown that an onset stimulus's success in capturing attention is contingent on top-down settings - in, for instance, Folk et al. (1992). It should be noted, however, that some more recent results have contradicted those of Folk et al. (e.g., Belopolsky, Schreij, \& Theeuwes, 2010; Lamy \& Egeth, 2003; Schreij, Owens, \& Theeuwes, 2008). Chua (submitted) also demonstrated bottom-up onset capture, but noted that the capture magnitude of the bottom-up onset capture effects was significantly smaller than in the condition with top-down modulation. Overall, the results of Experiments 1 and 2 suggest that the onset capture had been modulated by top-down mechanisms.
}

defined by several diagnostic features (e.g., "red" or "horizontal"). On any trial, some or all these features are found in the target. When the system is set to monitor the full set of target features, there would be a redundancy gain for trials in which the target contains all of the diagnostic features (the target is both "red" and "horizontal"), as compared to trials in which the target bears only a single feature (it is either "red" or "horizontal"; e.g., Krummenacher, Müller, \& Heller, 2001; Miller, 1982). The context of the experiments in this article was different. The target's configuration was entirely predictable, as all of its diagnostic features were always present. Therefore, monitoring the entire set of target features appears paradoxical: why should greater cognitive demands be put on the system when these added demands appear, at least on the surface, unlikely to yield dividends? In other words, can what is already an efficient search be facilitated further?

Evidence from the visual search literature has suggested that, in a singleton-search context, latencies were reliably shorter when a fixed target was used than when the target varied unpredictably (e.g., Bravo \& Nakayama, 1992; Wolfe, Butcher, Lee, \& Hyle, 2003). Wolfe et al. (2003) argued that when the singleton target's form is predictable, the visual detectors can be tuned to monitor, specifically, the fixed features. But, when the target's form is unpredictable, a wider bank of detectors will need to be deployed. Narrow tuning of the system's detectors allows the target's signal to register more strongly, which in turn facilitates information accumulation, and thereby improves response times.

Improving the time to locate the target was crucial to our task, as the observers were instructed to respond rapidly and the search letters were presented only for $200 \mathrm{~ms}$. To perform the task both accurately and quickly, it was imperative that the system deploy attention swiftly to the target location, so that the target letter could get adequately processed before it disappeared.

In the Wolfe et al. (2003) experiments, the contrast was between perfect prediction of the target singleton's form and imperfect prediction. In the experiments described in this article, the observers could always predict what the target would be. Thus, I argue that the same impetus of accumulating information quickly also applied in the present experimental context. Monitoring the most salient feature would, on average, lead to the target location most quickly. But, so long as there was variation in the time taken to locate the target, monitoring a single feature, even the most salient one, would not guarantee that the target would be reached fastest on all trials. We may think of this as three competing strategies of winning a horse race: One could (a) field a single fast horse that wins most, but not all, of the races; (b) field all of the horses in the stable; or (c) field only those horses that stand a chance of winning. Both strategies (b) and (c) guarantee winning all races, but (c) is the more 
economical strategy. The strategy adopted by the attention system is one that guarantees the fastest route to the target on all trials. To achieve this, the system monitors not all of the target's features, but only all of its unique features.

\section{Conclusion}

In a search scenario in which the target is embedded among heterogeneous distractors, the attentional system needs to be programmed to respond to the specific target features. However, in a search environment in which the target is a salient oddball, monitoring the complete inventory of target features may appear superfluous. The data suggest that the system tuned its detectors to monitor all the features that were diagnostic of targethood. Furthermore, as the target here was an oddball, the system included singleton detection as one aspect of its search strategy. In other words, the ACS is programmed with multiple, redundant triggers. The advantage is that attention can very swiftly hone in on the target, but with the disadvantage that when a set of features is prioritized, there could be inadvertent orienting to objects that possess some, but not all, of the prioritized features. The net result is a version of a speed-accuracy trade-off. When the premium is on locating the target swiftly, the system risks occasional capture by distractors.

Author note This research was supported by NUS Grant No. R-581000-136-112.

\section{References}

Anderson, B. A., \& Folk, C. L. (2010). Variations in the magnitude of attentional capture: Testing a two-process model. Attention, Perception, \& Psychophysics, 72, 342-352. doi:10.3758/ APP.72.2.342

Ansorge, U., \& Heumann, M. (2003). Top-down contingencies in peripheral cuing: The roles of color and location. Journal of Experimental Psychology. Human Perception and Performance, 29, 937-948. doi:10.1037/0096-1523.29.5.937

Bacon, W., \& Egeth, H. (1994). Overriding stimulus-driven attentional capture. Perception \& Psychophysics, 55, 485-496. doi:10.3758/ BF03205306

Belopolsky, A., Schreij, D., \& Theeuwes, J. (2010). What is top-down about contingent capture? Attention, Perception, \& Psychophysics, 72, 326-341. doi:10.3758/APP.72.2.326

Bravo, M. J., \& Nakayama, K. (1992). The role of attention in different visual-search tasks. Perception \& Psychophysics, 51, 465-472. doi:10.3758/BF03211642

Folk, C. L., \& Anderson, B. A. (2010). Target-uncertainty effects in attentional capture: Color-singleton set or multiple attentional control settings? Psychonomic Bulletin \& Review, 17, 421-426. doi:10.3758/PBR.17.3.421

Folk, C. L., \& Remington, R. (1998). Selectivity in distraction by irrelevant featural singletons: Evidence for two forms of attentional capture. Journal of Experimental Psychology. Human
Perception and Performance, 24, 847-858. doi:10.1037/00961523.24.3.847

Folk, C. L., \& Remington, R. W. (2008). Bottom-up priming of topdown attentional control settings. Visual Cognition, 16, 215-231. doi: $10.1080 / 13506280701458804$

Folk, C. L., Remington, R. W., \& Johnston, J. C. (1992). Involuntary covert orienting is contingent on attentional control settings. Journal of Experimental Psychology. Human Perception and Performance, 18, 1030-1044. doi:10.1037/0096-1523.18.4.1030

Folk, C. L., Remington, R. W., \& Wright, J. H. (1994). The structure of attentional control: Contingent attentional capture by apparent motion, abrupt onset, and color. Journal of Experimental Psychology. Human Perception and Performance, 20, 317-329. doi:10.1037/0096-1523.20.2.317

Irons, J. L., Folk, C. L., \& Remington, R. W. (2012). All set! evidence of simultaneous attentional control settings for multiple target colors. Journal of Experimental Psychology. Human Perception and Performance, 38, 758-775. doi:10.1037/a0026578

Itti, L., \& Koch, C. (2000). A saliency-based search mechanism for overt and covert shifts of visual attention. Vision Research, 40, 1489-1506. doi:10.1016/S0042-6989(99)00163-7

Jonides, J., \& Yantis, S. (1988). Uniqueness of abrupt visual onset in capturing attention. Perception \& Psychophysics, 43, 346-354. doi: $10.3758 / \mathrm{BF} 03208805$

Krummenacher, J., Müller, H. J., \& Heller, D. (2001). Visual search for dimensionally redundant pop-out targets: Evidence for parallelcoactive processing of dimensions. Perception \& Psychophysics, 63, 901-917. doi:10.3758/BF03194446

Lamy, D., Bar-Anan, Y., Egeth, H. E., \& Carmel, T. (2006a). Effects of top-down guidance and singleton priming on visual search. Psychonomic Bulletin \& Review, 13, 287-293. doi:10.3758/ BF03193845

Lamy, D., Carmel, T., Egeth, H. E., \& Leber, A. B. (2006b). Effects of search mode and intertrial priming on singleton search. Perception \& Psychophysics, 68, 919-932. doi:10.3758/ BF03193355

Lamy, D., \& Egeth, H. E. (2003). Attentional capture in singletondetection and feature-search modes. Journal of Experimental Psychology. Human Perception and Performance, 29, 10031020. doi:10.1037/0096-1523.29.5.1003

Lamy, D., Leber, A., \& Egeth, H. E. (2004). Effects of task relevance and stimulus-driven salience in feature-search mode. Journal of Experimental Psychology. Human Perception and Performance, 30, 1019-1031. doi:10.1037/0096-1523.30.6.1019

Lamy, D., \& Zoaris, L. (2009). Task-irrelevant stimulus salience affects visual search. Vision Research, 49, 1472-1480. doi:10.1016/ j.visres.2009.03.007

Maljkovic, V., \& Nakayama, K. (1994). Priming of pop-out: I. Role of features. Memory \& Cognition, 22, 657-672. doi:10.3758/ BF03209251

Miller, J. (1982). Divided attention: Evidence for coactivation with redundant signals. Cognitive Psychology, 14, 247-279. doi:10.1016/0010-0285(82)90010-X

Schreij, D., Owens, C., \& Theeuwes, J. (2008). Abrupt onsets capture attention independent of top-down control settings. Perception \& Psychophysics, 70, 208-218. doi:10.3758/PP.70.2.208

Theeuwes, J. (1992). Perceptual selectivity for color and form. Perception \& Psychophysics, 51, 599-606. doi:10.3758/ BF03211656

Theeuwes, J. (2010). Top-down and bottom-up control of visual selection. Acta Psychologica, 135, 77-99. doi:10.1016/ j.actpsy.2010.02.006

Todd, J. T., \& Van Gelder, P. (1979). Implications of a transientsustained dichotomy for the measurement of human performance. Journal of Experimental Psychology. Human Perception and Performance, 5, 625-638. doi:10.1037/0096-1523.5.4.625 
Treisman, A. M., \& Gelade, G. (1980). A feature-integration theory of attention. Cognitive Psychology, 12, 97-136. doi:10.1016/00100285(80)90005-5

Wolfe, J. M., Butcher, S. J., Lee, C., \& Hyle, M. (2003). Changing your mind: On the contributions of top-down and bottom-up guidance in visual search for feature singletons. Journal of Experimental Psychology. Human Perception and Performance, 29, 483-502. doi:10.1037/0096-1523.29.2.483

Wolfe, J. M., Cave, K. R., \& Franzel, S. L. (1989). Guided search: An alternative to the feature integration model for visual search. Journal of Experimental Psychology. Human Perception and Performance, 15, 419-433. doi:10.1037/0096-1523.15.3.419

Yantis, S., \& Egeth, H. E. (1999). On the distinction between visual salience and stimulus-driven attentional capture. Journal of
Experimental Psychology. Human Perception and Performance, 25, 661-676. doi:10.1037/0096-1523.25.3.661

Yantis, S., \& Hillstrom, A. P. (1994). Stimulus-driven attentional capture: Evidence from equiluminant visual objects. Journal of Experimental Psychology. Human Perception and Performance, 20, 95-107. doi:10.1037/0096-1523.20.1.95

Yantis, S., \& Jonides, J. (1984). Abrupt visual onsets and selective attention: Evidence from visual search. Journal of Experimental Psychology. Human Perception and Performance, 10, 601-621. doi:10.1037/0096-1523.10.5.601

Yantis, S., \& Jonides, J. (1996). Attentional capture by abrupt onsets: New perceptual objects or visual masking? Journal of Experimental Psychology. Human Perception and Performance, 22, 1505-1513. doi:10.1037/0096-1523.22.6.1505 